\title{
Performance of Oilseed Crops in Marathwada Region of Maharashtra State
}

\author{
S.S. Mulik*, S.S. More and R.D. Shelke \\ Department of Agricultural Economics \\ College of Agriculture, Latur, India \\ *Corresponding author
}

\section{A B S T R A C T}

\begin{tabular}{|l|}
\hline Ke y w or d s \\
$\begin{array}{l}\text { Growth, Oilseed, } \\
\text { Soybean, Safflower, } \\
\text { Marquardt } \\
\text { algorithm, Area, } \\
\text { Production and } \\
\text { productivity }\end{array}$ \\
\hline Article Info \\
\hline $\begin{array}{l}\text { Accepted: } \\
\text { 20 July } 2018 \\
\text { Available Online: } \\
\text { 10 August } 2018\end{array}$ \\
\hline
\end{tabular}

\section{Ke y w ords}

Growth, Oilseed, Marquardt algorithm, Area, Production and productivity

Article Info

Accepted:

20 July 2018

10 August 2018

\section{Introduction}

Agriculture has been and will continue to be the lifeline of the Indian economy. As the largest private enterprise in India, agriculture contributes nearly about 14 per cent of the national GDP, sustains livelihood of about two thirds of population and is the backbone of agro-based industries. A large number of important industries like textiles, vanaspati oils, jute, tobacco and sugar are sustained on raw materials produced in farm sector.
A research was conducted to assess the performance of oilseed crops in Marathwada region of Maharashtra State. Performance of oilseed crops was judged on important parameters i.e. growth. Compound growth rate was estimated by fitting non linear model to the area, production and productivity data for safflower from the period 1986-87 to 2015-16 i.e. 30 years. The entire period was divided in to three sub-periods and one overall period. Whereas for soybean crop because of non availability of data area, production and yield data were collected from 1996-97 to 2015-16 i.e. 20 year period. The entire period was divided in to two sub-periods and one overall period. The fitted model was analyzed using Marquardt algorithm. The results show that, Compound growth rate of area and production in soybean crop was positive and significant at district, region and state level during period I to period II and overall period except in Aurangabad district. In Aurangabad district compound growth rate of soybean was observed negatively during second period. Mixed trends were observed at districts, region and state in CGR of soybean productivity. The compound growth rate of area and production of safflower crop was negative and significant of many districts of Marathwada region. Mixed trend in CGR of safflower productivity was observed. 
Soybean (Glycine max) is important oil yielding rainy season crop having multiple uses. It stands second, among nine oilseed crops, next only to groundnut production in the country. It has outstanding nutritive value with $40-44 \%$ biological protein, $20 \%$ oil and is also very rich in vitamins, iron, mineral salts and amino acids. Soybean which is also known as soya beans are species of legume that have become one of the most widely consumed foods in the world. They are extremely useful for human health, and they are easy to cultivate as well. It was introduced into India in 1870-80.

Safflower (Carthamus tinctinctorious L.) belongs to family Asteraceae. It is one of the important oilseed crops and occupies second position among the major oilseed crops viz., groundnut, safflower, sesamum, mustard, linseed, and sunflower grown in India. It is important from the point of its economic importance due to high oil (24-36 per cent) and mineral value for human and cattle. In India, safflower as an oilseed crop introduced in 1970-71 onwards. It is one of the fastest growing crops in India.

Maharashtra is one of the leading oilseed growing states in India. Maharashtra State occupies a prominent place in the area and production of oilseed crops grown in our country. The oilseed crops can be grown in all kind of soils and constitutes an important part of crop rotation.

To achieve higher growth rates in oilseeds production and to narrow down the gaps between demands and supply the government of India launched from time to time number of production oriented programmes.

During 2015-16, all India estimated area, production and productivity of total oilseeds was 260.872 lakh ha, 252.508 lakh tonnes and $968 \mathrm{~kg}$ per ha, respectively. All India estimated area, production and productivity of soybean was 116.045 lakh ha, 85.698 lakh tonnes and $738 \mathrm{~kg}$ per ha, respectively. Area, production and productivity of safflower was 1.275 lakh ha, 0.33 lakh tonnes and $738 \mathrm{~kg}$ per ha, respectively. Major soybean growing states in India are Madhya Pradesh (58\%), Maharashtra (30\%), Rajasthan (6\%), Karnataka, Uttar Pradesh, Andhra Pradesh, Chhattisgarh and Gujarat.

In Marathwada region total area, production and yield under oilseed crops was 14.647 lakh ha, 4.453 tonnes and $304 \mathrm{~kg}$ per hectare respectively. Area, production and yield under soybean crop was 13.146 lakh ha, 4.167 lakh tonnes and $317 \mathrm{~kg}$ per hectare respectively. Area, production and yield under safflower crop was 510 ha, 0.111 lakh tonnes and 218 $\mathrm{kg}$ per hectare respectively.

\section{Objective}

1. To estimate growth rates of area, production and yield of major oilseed crops in Marathwada region.

\section{Materials and Methods}

\section{Data}

The data were collected from the Epitome of agriculture for the period of 30 years i.e. from 1986-87 to $2015-16$ for safflower. The entire study was split into three sub-periods and overall period. The sub-period was framed as Period I : : $1986-87$ to $1995-96$. Period II : : $1996-97$ to $2005-06$. Period Ш : : 2006-07 to 2015-16.

Overall Period : $1986-17$ to $2015-16$

Whereas for soybean crop because of non availability of data area, production and yield data were collected from 1996-97 to 2015-16 i.e. 20 year period. The entire period was 
divided in to two sub-periods and one overall period. The sub-period framed as

\section{Period I $\quad: 1996-97$ to 2005-06. \\ Period II : : 2006-07 to 2015-16.}

Overall Period : $1996-97$ to 2015-16.

\section{Analysis of data growth rate}

The compound growth rate was computed based on its fit using non -linear models, especially, the exponential model. Conventionally, the compound growth rates were estimated after converting the growth model to semi-log form and estimated through Ordinary Least Square (OLS) technique assuming multiplicative error term. However, there are several problems associated with this technique including the difficulty in estimating standard error of estimates of original parameters. Hence, a non-linear estimation technique for solving exponential model assuming additive error terms was used to estimate the compound growth rates.

$\mathrm{y}_{\mathrm{t}}=$ constant $*(1+\mathrm{CGR})^{\mathrm{t}}+\epsilon_{\mathrm{t}}$

Where,

$Y_{t}$ is the time series data for area/production/yield for year $t$, $t$ is the time trends for years of interest, $\epsilon_{\mathrm{t}}$ is the error term and

CGR is compound growth rate for the period under consideration.

The Marquardt algorithm will be used to estimate the parameters of equation. The data were smoothened by taking three year moving average to remove bias if any, induced by the outliers. The significance of regression coefficients will be tested by applying standard ' $\mathrm{t}$ ' test procedure.

\section{Results and Discussion}

\section{Soybean}

The annual compound growth rate (CGR) in area of soybean crops was $12.38,3.76$, and 7.92 per cent per annum during two subperiods and overall period at state level, respectively (Table 1). It was statistically significant at 1 per cent level of significance. This shows that, maximum growth in area of soybean crops has been occurred during first period (1996-97 to 2005-06). During second period, area under soybean crops was decreased (Except Aurangabad district). Area of soybean crops has shown positive and significant trend in all the three period of study but maximum growth was observed during first period. Under overall period, all districts of Marathwada region have exhibited positive growth rate in area of soybean. Highest growth rate observed in Osmanabad district (20.64) followed by Jalna (18.94 per cent) and Beed (15.18 per cent). All districts in Marathwada region were statistically significant at 1 per cent level of significance. The lowest positive compound growth rate in area of soybean was registered in Hingoli district 8.24 per cent per annum, which was found significant at 5 per cent level of significance. Growth rate under soybean area in Marathwada region, during the first period under study (1986-87 to 1995-96) i.e. 60.20 per cent, during sub sequent period it was 3.76, and 13.39 per cent i.e. Second and overall period, respectively.

Magnitude of CGR in regards to production of soybean was seen higher in first sub-period compared to second sub-period of study. The annual compound growth rate (CGR) in production of soybean crops was $10.68,2.03$, and 6.88 per cent per annum during two subperiods and overall period at state level, respectively (Table 1). Almost all districts in Marathwada region were statistically 
significant at 1 per cent level of significance (except Aurangabad district) during period first. This shows that, maximum growth in production of soybean crops has been occurred during first period (1996-97 to 200506). Non significant growth trends was observed during second period of study in Nanded and Hingoli and Maharashtra state Growth rate under soybean production in Marathwada region, during the first period under study (1996-97 to 2005-06) i.e. 53.32 per cent, during sub sequent period it was 10.22, and 13.61 per cent i.e. Second and overall period, respectively. Similar result was registered by Reddy and Immanuelraj (2017), in their studies.

The annual compound growth rate (CGR) in productivity of soybean crops was -0.46, 1.07 , and -0.82 per cent per annum during two sub-periods and overall period at state level, respectively (Table 1). Almost all districts in Marathwada region were statistically Nonsignificant (except Jalna district) during first and overall period. This shows that, maximum growth in productivity of soybean crops has been occurred during second period (2005-06 to 2015-16). During second period, productivity under soybean crops was increased except Aurangabad, Jalna, Parbhani and Hingoli districts. During overall period (1996-97 to 2015-16), It was observed that (Table 1) highest growth in productivity of soybean in Osmanabad district (3.42 per cent) and it was statistically significant at 5 per cent. Highest decline trend in productivity of soybean was registered in Nanded district was -2.32 per cent (with standard error 0.74). During first, second and overall period, productivity was statistically non-significant in Marathwada region. Growth rate under soybean productivity in Marathwada region, during the first period under study (1996-97 to 2005-06) i.e. 1.09 per cent, during subsequent period it was 4.38 , and 0.27 per cent i.e. Second and overall period, respectively.

\section{Safflower}

The results of growth analysis of area of safflower in Marathwada region and at Maharashtra state are presented in table 2. The annual compound growth rate (CGR) in area of safflower crops was $-2.30,-8.89$,11.56 and -5.04 per cent per annum during three sub-periods and overall period at state level, respectively (Table 1). Negative growth trends was observed almost all districts in Marathwada region except Nanded during period first and Hingoli during second and overall period of study. A highest decline trend in safflower area was observed during third period. During overall period (1986-87 to 2015-16), It was observed that (Table 2), positive growth in Hingoli district was 3.42 per cent and it was statistically nonsignificant. A highest decline trend in area of safflower was registered in Marathwada region during $3^{\text {rd }}$ period ( -9.78 per cent), but statistically it was significant at 1 per cent level. Growth rate under safflower area in Marathwada region during the first period under study (1986-87 to 1995-96) i.e. -0.84 per cent, during sub-sequent period it was 4.93, -9.78 and -3.09 per cent i.e. Second, third and overall period, respectively.

The annual compound growth rate (CGR) in production of safflower crops was 1.89, 11.86,-13.13 and -4.35 per cent per annum during three sub-periods and overall period at state level (Table 1). Negative growth trends was observed almost all districts in Marathwada region except Latur and Nanded districts during period first, Nanded and Hingoli districts during second and Hingoli district during overall period of study. Highest decline trend in safflower production was observed during third period. During overall period (1986-87), positive growth in Hingoli district was 4.02 per cent and it was statistically significant. 
Table.1 Growth in soybean area, production and yield in districts of Marathwada region and at Maharashtra state

\begin{tabular}{|c|c|c|c|c|c|c|c|c|c|c|}
\hline \multirow[t]{2}{*}{ Sr. No } & \multirow[t]{2}{*}{ Name } & \multicolumn{3}{|c|}{ Area } & \multicolumn{3}{|c|}{ Production } & \multicolumn{3}{|c|}{ Yield } \\
\hline & & Period-I & Period-II & Overall & Period-I & Period-II & Overall & Period-I & Period-II & Overall \\
\hline 01 & Aurangabad & $\begin{array}{l}6.78 * \\
(2.75)\end{array}$ & $\begin{array}{l}8.39 * \\
(3.67)\end{array}$ & $\begin{array}{c}9.79 * * \\
(1.38)\end{array}$ & $\begin{array}{l}6.91 * \\
(2.94)\end{array}$ & $\begin{array}{l}-5.14 * \\
(1.79)\end{array}$ & $\begin{array}{c}5.97 * * \\
(1.33)\end{array}$ & $\begin{array}{c}0.10^{\mathrm{NS}} \\
(0.94)\end{array}$ & $\begin{array}{c}-6.12^{*} \\
(1.92)\end{array}$ & $\begin{array}{c}-0.49^{\mathrm{NS}} \\
(0.70)\end{array}$ \\
\hline 02 & Jalna & $\begin{array}{c}33.16 * * \\
(6.61)\end{array}$ & $\begin{array}{c}16.49 * * \\
(1.14)\end{array}$ & $\begin{array}{c}18.94 * * \\
(0.82)\end{array}$ & $\begin{array}{c}39.74 * * \\
(6.22)\end{array}$ & $\begin{array}{l}9.59 * * \\
(2.28)\end{array}$ & $\begin{array}{c}14.84 * * \\
(1.78)\end{array}$ & $\begin{array}{c}6.44^{*} \\
(1.92)\end{array}$ & $\begin{array}{c}-1.73^{\mathrm{NS}} \\
(1.75)\end{array}$ & $\begin{array}{l}1.56 * \\
(0.76)\end{array}$ \\
\hline $\mathbf{0 3}$ & Beed & $\begin{array}{c}51.37 * * \\
(5.48)\end{array}$ & $\begin{array}{c}11.20 * * \\
(0.93)\end{array}$ & $\begin{array}{c}15.78 * * \\
(1.27)\end{array}$ & $\begin{array}{c}45.33 * * \\
(5.40)\end{array}$ & $\begin{array}{c}15.59 * \\
(6.06)\end{array}$ & $\begin{array}{c}17.19 * * \\
(3.26)\end{array}$ & $\begin{array}{l}1.81^{\mathrm{NS}} \\
(2.21)\end{array}$ & $\begin{array}{l}8.70^{\mathrm{NS}} \\
(5.07)\end{array}$ & $\begin{array}{c}0.29^{\mathrm{NS}} \\
(1.30)\end{array}$ \\
\hline 04 & Latur & $\begin{array}{c}79.67 * * \\
(8.25)\end{array}$ & $\begin{array}{c}8.77 * * \\
(0.34)\end{array}$ & $\begin{array}{c}13.78 * * \\
(1.53)\end{array}$ & $\begin{array}{c}88.70 * * \\
(7.31)\end{array}$ & $\begin{array}{l}16.06 * \\
(6.04)\end{array}$ & $\begin{array}{c}17.07 * * \\
(3.15)\end{array}$ & $\begin{array}{c}-0.70^{\mathrm{NS}} \\
(0.97)\end{array}$ & $\begin{array}{c}11.06^{\mathrm{NS}} \\
(5.29)\end{array}$ & $\begin{array}{l}2.90 * \\
(1.26)\end{array}$ \\
\hline 05 & Osmanabad & $\begin{array}{c}64.72 * * \\
(12.42)\end{array}$ & $\begin{array}{c}19.44 * * \\
(2.59)\end{array}$ & $\begin{array}{c}20.64 * * \\
(1.62)\end{array}$ & $\begin{array}{c}92.04 * * \\
(23.14)\end{array}$ & $\begin{array}{c}20.22 * \\
(6.13)\end{array}$ & $\begin{array}{c}21.31 * * \\
(3.56)\end{array}$ & $\begin{array}{c}0.67^{\mathrm{NS}} \\
(2.83)\end{array}$ & $\begin{array}{l}5.82^{N S} \\
(3.10)\end{array}$ & $\begin{array}{l}3.42 * \\
(1.00)\end{array}$ \\
\hline 06 & Nanded & $\begin{array}{c}65.11 * * \\
(2.71)\end{array}$ & $\begin{array}{l}3.78 * * \\
(0.62)\end{array}$ & $\begin{array}{c}11.93 * * \\
(1.75)\end{array}$ & $\begin{array}{c}51.80 * * \\
(4.24)\end{array}$ & $\begin{array}{l}5.29^{\mathrm{NS}} \\
(3.18)\end{array}$ & $\begin{array}{c}11.35 * * \\
(2.20)\end{array}$ & $\begin{array}{c}-2.69^{\mathrm{NS}} \\
(1.24)\end{array}$ & $\begin{array}{c}2.82^{\mathrm{NS}} \\
(3.10)\end{array}$ & $\begin{array}{c}-2.32 * * \\
(0.74)\end{array}$ \\
\hline 07 & Parbhani & $\begin{array}{c}38.50 * * \\
(2.08)\end{array}$ & $\begin{array}{c}9.05 * * \\
(0.66)\end{array}$ & $\begin{array}{c}13.79 * * \\
(1.05)\end{array}$ & $\begin{array}{c}29.09 * * \\
(2.91)\end{array}$ & $\begin{array}{l}4.27 * \\
(3.10)\end{array}$ & $\begin{array}{c}11.13 * * \\
(2.05)\end{array}$ & $\begin{array}{c}0.24^{\mathrm{NS}} \\
(1.60)\end{array}$ & $\begin{array}{c}-2.75^{\mathrm{NS}} \\
(1.98)\end{array}$ & $\begin{array}{c}-1.62 * \\
(0.65)\end{array}$ \\
\hline 08 & Hingoli & $\begin{array}{c}53.68 * * \\
(5.55)\end{array}$ & $\begin{array}{c}3.00 * * \\
(0.42)\end{array}$ & $\begin{array}{l}8.24 * * \\
(1.64)\end{array}$ & $\begin{array}{c}46.86 * * \\
(6.06)\end{array}$ & $\begin{array}{l}2.71^{\mathrm{NS}} \\
(3.30)\end{array}$ & $\begin{array}{l}7.22 * * \\
(2.22)\end{array}$ & $\begin{array}{c}-1.02^{\mathrm{NS}} \\
(1.25)\end{array}$ & $\begin{array}{c}-0.01^{\mathrm{NS}} \\
(2.97)\end{array}$ & $\begin{array}{c}-1.45^{\mathrm{NS}} \\
(0.89)\end{array}$ \\
\hline 09 & Marathwada region & $\begin{array}{c}60.20 * * \\
(3.85)\end{array}$ & $\begin{array}{c}8.21 * * \\
(0.40)\end{array}$ & $\begin{array}{c}13.39 * * \\
(1.35)\end{array}$ & $\begin{array}{c}53.32 * * \\
(3.57)\end{array}$ & $\begin{array}{l}10.22 * \\
(4.04)\end{array}$ & $\begin{array}{c}13.61 * * \\
(2.32)\end{array}$ & $\begin{array}{l}1.09^{N S} \\
(0.94)\end{array}$ & $\begin{array}{c}4.38^{\mathrm{NS}} \\
(3.21)\end{array}$ & $\begin{array}{c}0.27^{\mathrm{NS}} \\
(0.77)\end{array}$ \\
\hline 10 & Maharashtra State & $\begin{array}{c}12.38 * * \\
(1.29)\end{array}$ & $\begin{array}{c}3.76 * * \\
(0.39)\end{array}$ & $\begin{array}{l}7.92 * * \\
(0.62)\end{array}$ & $\begin{array}{c}10.68 * * \\
(1.00)\end{array}$ & $\begin{array}{c}2.03^{N S} \\
(2.17)\end{array}$ & $\begin{array}{c}6.88 * * \\
(1.03)\end{array}$ & $\begin{array}{c}-0.46^{\mathrm{NS}} \\
(0.58)\end{array}$ & $\begin{array}{c}-1.07^{\mathrm{NS}} \\
(2.21)\end{array}$ & $\begin{array}{c}-0.82^{\mathrm{NS}} \\
(0.52)\end{array}$ \\
\hline
\end{tabular}

Note: Period-I: 1996-97 to 2005-06, Period-II: 2006-07 to 2015-16, Overall Period: 1996-97 to 2015- 16. Figures in bracket indicate standard error,* denotes significant at 5 percent, $* *$ denotes significant at 1 percent, NS-Non Significant 
Table.2 Growth in safflower area, production and yield in districts of Marathwada region and at Maharashtra state

\begin{tabular}{|c|c|c|c|c|c|c|c|c|c|c|c|c|c|}
\hline \multirow{3}{*}{$\begin{array}{l}\text { Sr. } \\
\text { No } \\
01\end{array}$} & \multirow{3}{*}{$\begin{array}{c}\text { Name } \\
\text { Aurangabad }\end{array}$} & \multicolumn{4}{|c|}{ Area } & \multicolumn{4}{|c|}{ Production } & \multicolumn{4}{|c|}{ Yield } \\
\hline & & \multirow{2}{*}{$\begin{array}{l}\text { Period-I } \\
6.52 * * \\
(1.53)\end{array}$} & \multirow{2}{*}{\multicolumn{2}{|c|}{$\begin{array}{l}\text { Period-II Period-III } \\
\left.\begin{array}{c}-11.77 * *) \\
(0.82)\end{array}\right)(2.87 * * \\
\end{array}$}} & \multirow{2}{*}{$\begin{array}{l}\text { Overall } \\
\begin{array}{c}-8.12 * * \\
(0.50)\end{array}\end{array}$} & \multirow{2}{*}{\begin{tabular}{|c|} 
Period-I \\
$-3.78^{\mathrm{NS}}$ \\
$(3.44)$
\end{tabular}} & \multirow{2}{*}{\begin{tabular}{|c|} 
Period-II \\
$-16.18 * *$ \\
$(2.79)$
\end{tabular}} & \multirow{2}{*}{$\begin{array}{l}\text { Period- } \\
\text { III } \\
-25.57 * * \\
(2.24)\end{array}$} & \multirow{2}{*}{$\begin{array}{l}\text { Overall } \\
\begin{array}{c}-7.09 * * \\
(0.92)\end{array}\end{array}$} & \multirow{2}{*}{\begin{tabular}{|l|} 
Period-I \\
$1.94^{\mathrm{NS}}$ \\
$(2.04)$
\end{tabular}} & \multicolumn{2}{|c|}{$\begin{array}{c}\text { Period-II Period- } \\
\text { III }\end{array}$} & \multirow{2}{*}{$\begin{array}{c}\text { Overall } \\
\begin{array}{c}3.19 * * \\
(0.56)\end{array}\end{array}$} \\
\hline & & & & & & & & & & & $\begin{array}{c}-0.51^{\mathrm{NS}} \\
(2.87)\end{array}$ & $\begin{array}{l}-0.11^{\mathrm{NS}} \\
(1.72)\end{array}$ & \\
\hline 02 & Jalna & $\begin{array}{l}4.49 * * \\
(0.96)\end{array}$ & $\begin{array}{c}-10.17 * *) \\
(0.80)\end{array}$ & $\begin{array}{l}1.27 * * \\
(2.46)\end{array}$ & $\begin{array}{c}-6.77 * * \\
(0.43)\end{array}$ & $\begin{array}{c}-2.07^{\mathrm{NS}} \\
(1.76)\end{array}$ & $\begin{array}{c}-1.49^{\mathrm{NS}} \\
(2.36)\end{array}$ & $\begin{array}{c}-23.16 * * \\
(1.92)\end{array}$ & $\begin{array}{c}-5.08 * * \\
(0.63)\end{array}$ & $\begin{array}{l}3.47^{*} \\
(1.56)\end{array}$ & $\begin{array}{c}11.06 * * \\
(1.84)\end{array}$ & $\begin{array}{c}-0.34^{\mathrm{NS}} \\
(2.05)\end{array}$ & $\begin{array}{l}2.74 * * \\
(0.50)\end{array}$ \\
\hline 03 & Beed & $\begin{array}{l}6.98 * * \\
(1.29)\end{array}$ & $\begin{array}{c}-2.50 * * ? \\
(0.48)\end{array}$ & $\begin{array}{l}3.03 * * \\
(1.87)\end{array}$ & $\begin{array}{c}-5.24 * * \\
(0.51)\end{array}$ & $\begin{array}{c}-4.30^{\mathrm{NS}} \\
(3.06)\end{array}$ & $\begin{array}{l}-6.02 * \\
(2.22)\end{array}$ & $\begin{array}{c}-24.43 * * \\
(1.66)\end{array}$ & $\begin{array}{c}-5.91 * * \\
(0.71)\end{array}$ & $\begin{array}{l}2.66^{\mathrm{NS}} \\
(2.39)\end{array}$ & $\begin{array}{c}-3.81^{\mathrm{NS}} \\
(2.17)\end{array}$ & $\begin{array}{l}2.86^{\mathrm{NS}} \\
(2.31)\end{array}$ & $\begin{array}{c}-0.01^{\mathrm{NS}} \\
(0.47)\end{array}$ \\
\hline 04 & Latur & $\begin{array}{l}-2.54^{\mathrm{NS}} \\
(2.65)\end{array}$ & $\begin{array}{c}-5.93 * * ? \\
(1.35)\end{array}$ & $\begin{array}{l}2.87 * * \\
(2.68)\end{array}$ & $\begin{array}{c}-3.37 * * \\
(0.79)\end{array}$ & $\begin{array}{l}6.50^{\mathrm{NS}} \\
(3.98)\end{array}$ & $\begin{array}{l}-9.22 * \\
(3.01)\end{array}$ & $\begin{array}{c}-25.57 * * \\
(2.24)\end{array}$ & $\begin{array}{c}-1.60^{\mathrm{NS}} \\
(0.98)\end{array}$ & $\begin{array}{c}10.22 * * \\
(1.97)\end{array}$ & $\begin{array}{c}-0.11^{\mathrm{NS}} \\
(2.31)\end{array}$ & $\begin{array}{c}-0.10^{\mathrm{NS}} \\
(1.72)\end{array}$ & $\begin{array}{l}4.01 * * \\
(0.50)\end{array}$ \\
\hline 05 & Osmanabad & $\begin{array}{l}1.52^{\mathrm{NS}} \\
(1.39)\end{array}$ & $\begin{array}{c}-1.86 * * \\
(0.48)\end{array}$ & $\begin{array}{l}4.33 * * \\
(0.27)\end{array}$ & $\begin{array}{c}-1.60 * * \\
(0.21)\end{array}$ & $\begin{array}{c}-0.62^{\mathrm{NS}} \\
(2.35)\end{array}$ & $\begin{array}{c}-4.76^{N S} \\
(2.54)\end{array}$ & $\begin{array}{c}-7.94 * * \\
(0.75)\end{array}$ & $\begin{array}{c}-1.31 * * \\
(0.46)\end{array}$ & $\begin{array}{l}0.03^{\mathrm{NS}} \\
(1.70)\end{array}$ & $\begin{array}{c}-2.20^{\mathrm{NS}} \\
(2.29)\end{array}$ & $\begin{array}{c}-4.39 * * \\
(0.86)\end{array}$ & $\begin{array}{l}0.29^{\mathrm{NS}} \\
(0.39)\end{array}$ \\
\hline 06 & Nanded & $\begin{array}{l}3.53 * * \\
(0.88)\end{array}$ & $\begin{array}{c}-4.85 * * \\
(1.17)\end{array}$ & $\begin{array}{l}8.21 * * \\
(0.74)\end{array}$ & $\begin{array}{c}-1.18 * \\
(0.46)\end{array}$ & $\begin{array}{l}0.31^{\mathrm{NS}} \\
(2.83)\end{array}$ & $\begin{array}{l}0.25^{\mathrm{NS}} \\
(2.82)\end{array}$ & $\begin{array}{c}-8.38 * * \\
(1.56)\end{array}$ & $\begin{array}{l}-1.16^{*} \\
(0.51)\end{array}$ & $\begin{array}{c}-2.53^{\mathrm{NS}} \\
(1.87)\end{array}$ & $\begin{array}{l}4.66 * \\
(1.87)\end{array}$ & $\begin{array}{c}-2.22^{\mathrm{NS}} \\
(1.61)\end{array}$ & $\begin{array}{l}0.11^{\mathrm{NS}} \\
(0.36)\end{array}$ \\
\hline 07 & Parbhani & $\begin{array}{l}1.26^{\mathrm{NS}} \\
(0.99)\end{array}$ & $\begin{array}{c}-11.62 * * \\
(1.99)\end{array}$ & $\begin{array}{l}8.91 * * \\
(1.89)\end{array}$ & $\begin{array}{c}-4.16 * * \\
(0.44)\end{array}$ & $\begin{array}{c}-0.82^{\mathrm{NS}} \\
(2.14)\end{array}$ & $\begin{array}{c}-10.48 * \\
(3.51)\end{array}$ & $\begin{array}{c}-11.79 * * \\
(1.81)\end{array}$ & $\begin{array}{c}-4.41 * * \\
(0.58)\end{array}$ & $\begin{array}{c}-2.14^{\mathrm{NS}} \\
(1.44)\end{array}$ & $\begin{array}{l}2.16^{\mathrm{NS}} \\
(2.67)\end{array}$ & $\begin{array}{c}-5.09 * * \\
(0.82)\end{array}$ & $\begin{array}{c}-0.66^{\mathrm{NS}} \\
(0.35)\end{array}$ \\
\hline 08 & Hingoli & NA & $\begin{array}{l}7.38 * * \\
(0.53)\end{array}$ & $\begin{array}{l}6.46^{* *} \\
(1.15)\end{array}$ & $\begin{array}{c}0.15^{\mathrm{NS}} \\
(0.90)\end{array}$ & NA & $\begin{array}{c}14.59 * * \\
(1.00)\end{array}$ & $\begin{array}{c}-5.49 * * \\
(1.47)\end{array}$ & $\begin{array}{l}4.02 * \\
(1.46)\end{array}$ & NA & $\begin{array}{l}6.90 * * \\
(0.88)\end{array}$ & $\begin{array}{l}3.59^{\mathrm{NS}} \\
(2.65)\end{array}$ & $\begin{array}{c}4.59 * * \\
(0.96)\end{array}$ \\
\hline 09 & $\begin{array}{l}\text { Marathwada } \\
\text { region }\end{array}$ & $\begin{array}{l}0.84^{\mathrm{NS}} \\
(1.62)\end{array}$ & $\begin{array}{c}-4.93 * * \\
(0.95)\end{array}$ & $\begin{array}{l}9.78 * * \\
(0.30)\end{array}$ & $\begin{array}{c}-3.09 * * \\
(0.33)\end{array}$ & $\begin{array}{c}-1.69^{\mathrm{NS}} \\
(2.22)\end{array}$ & $\begin{array}{c}-5.18^{\mathrm{NS}} \\
(2.69)\end{array}$ & $\begin{array}{c}-11.13 * * \\
(0.61)\end{array}$ & $\begin{array}{c}-3.21 * * \\
(0.46)\end{array}$ & $\begin{array}{l}1.29^{\mathrm{NS}} \\
(1.43)\end{array}$ & $\begin{array}{c}-1.47^{\mathrm{NS}} \\
(2.69)\end{array}$ & $\begin{array}{l}-2.41 * \\
(0.92)\end{array}$ & $\begin{array}{l}0.32 * \\
(0.34)\end{array}$ \\
\hline 10 & $\begin{array}{l}\text { Maharashtra } \\
\text { State }\end{array}$ & $\begin{array}{l}-2.30 * \\
(0.78)\end{array}$ & $\begin{array}{c}-8.89 * * \\
(0.68)\end{array}$ & $\begin{array}{l}-11.56 \\
(0.40)\end{array}$ & $\begin{array}{c}-5.04 * * \\
(0.31)\end{array}$ & $\begin{array}{l}1.89^{\mathrm{NS}} \\
(1.92)\end{array}$ & $\begin{array}{c}-11.86 * * \\
(2.41)\end{array}$ & $\begin{array}{c}-13.13 * * \\
(0.67)\end{array}$ & $\begin{array}{c}-4.35 * * \\
(0.59)\end{array}$ & $\begin{array}{l}4.13 * \\
(1.33)\end{array}$ & $\begin{array}{c}-1.35^{\mathrm{NS}} \\
(1.69)\end{array}$ & $\begin{array}{c}-2.99 * * \\
(0.74)\end{array}$ & $\begin{array}{l}0.68 * \\
(0.29)\end{array}$ \\
\hline
\end{tabular}

Note: Period-I: 1986-87 to 1995-96, Period-II: 1996-97 to 2005-06, Period-III: 2006-07 to 2015-16, Overall Period: 1986-87 to 2015- 16.

Figures in bracket indicate standard error, ${ }^{*}$ denotes significant at 5 percent, ** denotes significant at 1 percent, NS - Non Significant 
During overall period, the compound growth rates of production of safflower registered negative growth in all districts except in Hingoli which shows positive growth (4.02 per cent) and statistically significant at 5 per cent level. Production under safflower crop in Marathwada region was negative in growth and statistically non-significant during $1^{\text {st }}$ and $2^{\text {nd }}$ period. Growth rate under safflower production in Marathwada region during the first period under study (198687 to $1995-96)$ i.e. -1.69 per cent, during subsequent period it was $-5.18,-11.13$ and -3.21 per cent i.e. Second, third and overall period, respectively.

The annual compound growth rate (CGR) in productivity of safflower crops was $4.13,-1.35$,2.99 and -0.68 per cent per annum during three sub-periods and overall period at state level, (Table 1). Positive growth trends was observed almost all districts in Marathwada region except Nanded and Parbhani districts during period first, Beed and Parbhani district during overall period of study. Highest decline trend in safflower productivity was observed during second and third period. During overall period, the compound growth rates of productivity of safflower registered positive growth in all districts except in Beed and Parbhani districts which shows negative growth and statistically non-significant. Positive and significant growth in safflower productivity was observed during overall period in Marathwada region. Highest growth rate was observed in productivity of safflower in Maharashtra state during period first.

In conclusion, it was observed that, average area and average production under soybean in all districts Marathwada region and at Maharashtra level was increased in sub-sequenced period, it was the proof of the fact that the soybean crop was a traditional crop in the region as well as the state as a whole. Hence there is very big need to concentrate on this crop for policy maker and researcher. Study concluded that soybean which is major oilseed crop expanded and stable in the region but the productivity levels are continuously reduced. Safflower which is the second major oilseed crop of the region lost substantial area from the cropping system. Hence proper attention may give to sustain and enhance the productivity level of soybean. Secondly proper incentive may give to safflower farmers, so as to cultivate this low productive crop in the region.

\section{References}

Anonymous (1981-2000), District wise General Statistical Information of Agriculture Department, Part II- Epitome of Agricultural in Maharashtra. Commission orate of Agricultural, Government of Maharashtra.

Anonymous (2016), Agricultural Statistics at a Glance, Ministry of Agriculture and farmer's welfare, Department of Agriculture, cooperation and farmer's welfare, Directorate of Agriculture Economics and Statistics.

More, S.S., Narendra Singh and Bhatt, B.K. (2018), Performance of pulses in Gujarat: A district level Assessment, Current Agriculture Research Journal, 6(1): 45-53.

Agarwal, P.K., Divya, P., Yadav, P., Sing, O.P., (2014), Trends of area, production and productivity of soybean crops in Madhya Pradesh, National Academy of Agricultural Science, 32(3-4): 797-800.

Datarkar, S., Pagire, B.V., Darekar, A., and Hile, R.B. (2015), Region-wise compound growth rates in area, production \& productivity of kharif groundnut in Maharashtra, International Journal of Tropical Agricultural (C) serial publication, 33(2):

\section{How to cite this article:}

Mulik, S.S., S.S. More and Shelke, R.D. 2018. Performance of Oilseed Crops in Marathwada Region of Maharashtra State. Int.J.Curr.Microbiol.App.Sci. 7(08): 3663-3669.

doi: https://doi.org/10.20546/ijcmas.2018.708.371 